Revue internationale P.M.E.

\title{
Les net-up... une nouvelle forme du projet entrepreneurial ?
}

Caractérisation du concept de net-up au travers de sa

communauté et de ses pratiques de cocréation

\section{The net-up... a new form of entrepreneurial project ?}

Characterization of the concept of net-up through its

community and co-creation practices

\section{La net-up... una nueva forma de proyecto empresarial ?}

Caracterización del concepto de la net-up a través de su

comunidad y su práctica de la co-creación

\section{Gaëlle Dechamp et Émilie Vez}

Volume 30, numéro 2, 2017

URI : https://id.erudit.org/iderudit/1040456ar

DOI : https://doi.org/10.7202/1040456ar

Aller au sommaire du numéro

\section{Éditeur(s)}

Editions EMS - In Quarto SARL

\section{ISSN}

0776-5436 (imprimé)

1918-9699 (numérique)

Découvrir la revue

Citer cet article

Dechamp, G. \& Vez, É. (2017). Les net-up... une nouvelle forme du projet entrepreneurial ? Caractérisation du concept de net-up au travers de sa communauté et de ses pratiques de cocréation. Revue internationale P.M.E., 30(2), 61-85. https://doi.org/10.7202/1040456ar
Résumé de l'article

Quid de la dimension communautaire dans la création de start-up ? La notion de net-up, projet entrepreneurial démarré grâce à l'appui d'une communauté, répond en partie à cette interrogation. Notre recherche, de nature exploratoire, consiste en l'approfondissement des concepts propres à la net-up, selon la définition proposée par Burret (2012). Plus spécifiquement, il s'agira de mettre en évidence les caractéristiques de la communauté regroupée autour de ces projets entrepreneuriaux et d'étudier les mécanismes à la base d'une dynamique cocréative entre un porteur de projet et une communauté présente dans un tiers-lieu. 


\title{
Les net-up... une nouvelle forme du projet entrepreneurial ? Caractérisation du concept de net-up au travers de sa communauté et de ses pratiques de cocréation
}

\author{
Gaëlle DECHAMP
}

Gaëlle Dechamp est maître de conférences en sciences de gestion à l'Université de Lyon, site de Saint-Étienne. Elle est chercheure titulaire du laboratoire CoActiS (conception de l'action en situation). Ses centres d'intérêt couvrent l'intégration des pratiques de créativité (notamment le design) dans les PME, les pratiques pédagogiques créatives ainsi que les modalités de développement des territoires créatifs (entrepreneuriat créatif, tiers-lieux...). Elle est responsable du master "prospective design " à Saint-Étienne, coopéré entre l'IAE, l'École nationale des mines et l'École supérieure d'art et de design. Elle est également responsable adjointe du projet Fabrique de l'Innovation de l'Université de Lyon.

Université Jean Monnet de Saint-Étienne

CoActiS

42023 SAINT-ÉTIENNE CEDEX 2, France gaelle.dechamp@univ-st-etienne.fr

Émilie VEZ

Émilie Vez est diplômée de HEC de l'Université de Genève et est titulaire d'un master en management du design et de l'innovation délivré conjointement par trois institutions de Saint-Étienne : l'École nationale des mines, l'École supérieure d'art et de design et l'IAE. Actuellement impliquée dans le déploiement d'un programme d'innovation ouverte dans une entreprise pharmaceutique internationale, Émilie accompagne à la création de projets d'innovation technologique, sociale et économique au sein de lieux d'innovation ouverts de type espaces de coworking, fablabs, bio-hackerspaces et autres tiers-lieux.

177 rue du Leman,

74140 CHENS SUR LEMAN, France emilie.vez@gmail.com

\section{RÉSUMÉ}

Quid de la dimension communautaire dans la création de start-up ? La notion de net-up, projet entrepreneurial démarré grâce à l'appui d'une communauté, répond en partie à cette interrogation. Notre recherche, de nature exploratoire, consiste en l'approfondissement des concepts propres à la netup, selon la définition proposée par Burret (2012). Plus spécifiquement, il s'agira de mettre en évidence les caractéristiques de la communauté regroupée autour de ces projets entrepreneuriaux et d'étudier les mécanismes à la base d'une dynamique cocréative entre un porteur de projet et une communauté présente dans un tiers-lieu.

MOTS-CLÉS

Net-up, Cocréation, Tiers-lieux, Start-up 


\title{
The net-up... a new form of entrepreneurial project? Characterization of the concept of net-up through its community and co-creation practices
}

\begin{abstract}
What about the Community dimension in the creation of start-up ? The notion of net-up, an entrepreneurial project started with the support of a community, partially answered this question. Our exploratory research, consists in deepening the concepts of net-up, according to the definition proposed by Burret (2012). More specifically, we highlight the characteristics of the community grouped around these entrepreneurial projects and also study the mechanisms underlying a co-creative dynamic between a project leader and a community present in a third place.
\end{abstract}

KEYWORDS

Net-up, Co-creation, Third place, Start-up

\section{La net-up... una nueva forma de proyecto empresarial ? Caracterización del concepto de la net-up a través de su comunidad y su práctica de la co-creación}

\begin{abstract}
RESUMEN
Que pasa con la dimensión comunitaria en la creación de start-up ? El concepto de net-up, proyecto empresarial que se inició con el apoyo de una comunidad, ha respondido parcialmente a esta pregunta. Nuestra investigación, de carácter exploratorio, consiste en la profundización de los conceptos específicos de la net-up, de acuerdo con la definición propuesta por Burret (2012). Más específicamente, se hará hincapié en las características de la comunidad agrupados en torno a estos proyectos empresariales y en el estudio de los mecanismos subyacentes a una dinámica co-creativa entre el lider de un proyecto y una comunidad presente en un tercio lugar.
\end{abstract}

Palabras clave

Net-up, Co-creación, Tercio lugar, Start-up

\section{INTRODUCTION}

La start-up constitue sans doute l'un des derniers espaces qui véhicule le mythe de l'entrepreneuriat héroïque : moteur, puissant, capable de retourner les forces en présence à son avantage (Murnieks, Cardon, Sudek, White et Brooks, 2016) - et pourtant son environnement semble standardisé : business plan, capital-risque, cluster, incubateur... Or cet environnement est en pleine mutation, en raison de l'essor, entre autres, des réseaux sociaux, de la pratique répandue des équipes entrepreneuriales dans les start-up et des lieux de travail alternatifs (tiers-lieux, coworking...). Quid de la dimension communautaire des projets de type start-up ? Le succès pour un entrepreneur dépend souvent de la dynamique sociale du projet, c'est-à-dire de sa capacité à fédérer un nombre croissant de parties prenantes. Cette pratique émergente qui est peu présente dans la littérature scientifique en entrepreneuriat, 
concerne une modalité spécifique de conception de projets entrepreneuriaux. En 2010, elle apparait pour la première fois dans la littérature sous l'appellation de «net-up » qui qualifie les nouvelles pratiques issues de la création collective (Comtesse et Pauletto, 2010). Par analogie avec la start-up, la net-up consiste en un projet ou une entreprise visant une croissance rapide (up), en mettant au cœur de son développement la communauté (net). Le tiers-lieu ${ }^{1}$ en est le lieu privilégié de développement en favorisant l'apprentissage collectif par la collaboration entre les entrepreneurs (partage des équipements, des connaissances et des valeurs par un réseau de sociabilité). Sont qualifiés de net-up des projets entrepreneuriaux qui répondent à trois critères : un projet porté par un individu qui prend racine au sein d'un tiers-lieu ; qui se développe par, autour et avec une communauté ; qui engendre des pratiques de cocréation (Burret, 2012). Cette approche étant en émergence, notre travail est une recherche de nature exploratoire, dont l'objectif consiste en l'approfondissement des concepts propres à la net-up. Nous souhaitons contribuer à la théorisation de cette nouvelle pratique entrepreneuriale, en nous concentrant sur un aspect selon nous fondamental : la cocréation. C'est une pratique spécifique intégrant des communautés de consommateurs dans un modèle dit de "l'innovation ouverte ». Plus spécifiquement, il s’agira (1) de mettre en évidence les caractéristiques de la communauté regroupée autour de ces projets et (2) d'étudier les mécanismes à la base d'une dynamique cocréative entre un porteur de projet et une communauté présente dans un tiers-lieu.

Le cadre théorique proposé se centre sur le phénomène de cocréation. C'est en effet la collaboration active d'une communauté autour du porteur de projet, et donc la cocréation qui en résulte, qui permet le développement de la net-up. Or cette dimension entrepreneuriale est peu étudiée en profondeur dans les travaux sur les start-up. Deux perspectives de la cocréation seront présentées afin de permettre l'analyse des pratiques des net-up. La première reprend les typologies des formes sociales communautaires que l'on retrouve dans les recherches en innovation ouverte et la seconde met en perspective la cocréation présente dans les projets de net-up avec le modèle de l'open source. Suite à l'énoncé de la problématique et à la présentation de la méthodologie, une troisième partie présentera les résultats basés sur une étude approfondie de type ethnographique, de deux projets qui se sont créés et développés dans des tiers-lieux. Nos résultats montrent principalement que le développement de la net-up s'appuie sur une forme hybride de communautés et qu'elle nécessite une vision élargie de la propriété intellectuelle. Notre discussion est finalement centrée sur trois thèmes principaux : les différents types de tiers-lieux, la construction d'un commun et la forme des communautés.

\section{CADRE THÉORIQUE}

Les origines du concept de cocréation se situent dans deux domaines spécifiques, ce qui ne facilite pas la compréhension des net-up (Stevens, 2009). D’une part, abordée sous l'angle du marketing de service, la cocréation se situe dans la lignée de la littérature portant sur les pratiques d'ouverture des activités innovantes des entreprises (innovation ouverte). D’autre

1 Cf. glossaire. 
part, la cocréation est en partie inspirée des pratiques informatiques de l'open source (Von Hippel, 1986). La première partie de notre cadre théorique s'attache à comprendre les différentes communautés identifiées dans les recherches sur la cocréation, et plus généralement en innovation ouverte. Puis, dans la mesure où les net-up se développent au sein de tierslieux, nous nous intéresserons aux pratiques issues de l'open source. En effet, le porteur du projet de net-up et la communauté qu'il agrège sont soumis à des règles et une philosophie imposées par le manifeste des tiers-lieux, lui-même issu de l'open source. La deuxième partie du cadre théorique proposera une synthèse de ces travaux sous la forme d'une grille d'analyse et formulera la problématique de la recherche.

\subsection{Caractérisation des communautés mobilisées dans les pratiques de cocréation}

Chesbrough (2003) pose les contours d'un modèle d'innovation dite " ouverte », en opposition à l'innovation fermée (conduite en interne à l'entreprise). Dans son modèle, les entreprises doivent capitaliser sur les connaissances développées à l'extérieur de leur structure pour stimuler l'innovation technologique et rentabiliser les connaissances développées en interne. L'idée d'ouverture est caractérisée par toutes formes de coopération externe à l'entreprise avec tous types de partenaires (concurrents, fournisseurs, utilisateurs, clients, etc.). Dans la lignée, Prahalad et Ramaswamy (2004) suggèrent qu'il est nécessaire de cocréer la valeur avec les consommateurs. Deux stratégies de cocréation avec les consommateurs sont possibles à mettre en œuvre (Hamdi-Kidar, 2013) : une stratégie dite « sélective » qui consiste à cibler les consommateurs les plus créatifs et innovants - dit lead users (Bécheur et Gollety, 2006), et une stratégie dite « extensive» dans laquelle tous les consommateurs peuvent participer (crowdsourcing) (Burger-Helmchen et Pénin, 2011 ; Ruiz, Brion et Parmentier, 2015).

Au-delà de la mobilisation des consommateurs et pour caractériser plus globalement les communautés à l'œuvre dans les pratiques d'innovation ouverte et de cocréation, l'un des cadres théoriques régulièrement utilisés est celui de la communauté de pratique (Wenger, 1998 ; Elliott et Scacchi, 2003 ; Madanmohan et Navelkar, 2002). Pour Wenger (1998), «communities of practice structure an organization's learning potential in two ways: through the knowledge they develop at their core and through interactions at their boundaries". Chanal (2000) explique que la notion de pratique se comprend au sens du "faire ", c'est-à-dire la capacité des individus à donner du sens aux actions par la production. La pratique englobe à la fois l'explicite (langage, outils, documents, règles, dont la pratique rend explicite) et l'implicite (des représentations, des conventions, etc.). Associée au concept de communauté, la pratique s'envisage alors comme la "source de cohérence " d'un groupe de personnes (Chanal, 2000, p. 7). En résumé, les communautés de pratique peuvent se définir en tant que " groupes d'individus qui ont une histoire commune, interagissent fréquemment, partagent des connaissances et rencontrent des problèmes proches, au sein d'une même organisation". Surtout, «ils travaillent ensemble et réalisent des activités pour partie communes, pour partie complémentaires» (Vaast, 2002, p. 3).

Pour Cohendet, Creplet et Dupouët (2003), les communautés de pratique ont pour objectif la réussite d'une activité alors que les communautés épistémiques s'orientent particulièrement 
vers la création et l'acquisition de nouvelles connaissances. Pour atteindre cette finalité, les membres de la communauté se soumettent à une certaine forme d'autorité régie par des procédures. Ces procédures sont un ensemble de règles ou de codes de bonne conduite, tacites ou explicites. Elles définissent les objectifs ainsi que les moyens à mettre en ouvre pour les atteindre, et régissent les comportements collectifs au sein de la communauté. Les communautés épistémiques sont donc structurées autour d'un but à atteindre (création et acquisition de connaissances partagées) et d'une autorité procédurale fondée, ou tout du moins validée par elles-mêmes. Dans cette configuration, la finalité d'acquisition de connaissance se place au-dessus des objectifs individuels, contrairement aux communautés de pratique qui placent l'autonomie et l'identité des individus au centre. Ainsi, Cohendet, Creplet et Dupouët (2003, p. 105) définissent les communautés épistémiques en tant que "groupe de représentants partageant un objectif cognitif commun de création de connaissance et une structure commune permettant une compréhension partagée ».

La caractérisation de la communauté mobilisée autour du porteur de projet est essentielle afin de mieux comprendre les net-up. Cependant en raison de la philosophie particulière des tiers-lieux (Burret, 2014, 2015) dans lesquels elles se développent, il importe de compléter ces approches classiques des communautés par un travail sur la dimension libre, fondatrice de l'open source.

Initialement, l'open source fait référence à une gestion particulière de la propriété intellectuelle appliquée à un logiciel informatique. Ce principe dit du copyleft s'appuie sur les fondements du droit d'auteur, mais au lieu de viser à restreindre les libertés des utilisateurs comme le copyright, le copyleft incite les auteurs à accorder aux utilisateurs plus de liberté (le terme "open source " a été formalisé en 1998).

Le développement d'un logiciel sous licence libre ${ }^{2}$ sappuie sur une communauté de développeurs/utilisateurs. En effet, par le biais des licences et en donnant accès au code source, l'entreprise incite des programmeurs à former une communauté intéressée à développer, modifier ou tester le logiciel (Ayerbe et Chanal, 2011). L'intérêt pour l'entreprise est de bénéficier d'une amélioration continue de son produit, tout en minimisant les investissements financiers. On reconnaît ici les fondements des modèles des utilisateurs experts (l'appropriation et l'amélioration d'un produit par un groupe d'utilisateurs experts, ici assimilé à la communauté de développeurs) et du crowdsourcing. Loilier et Tellier $(2004,2011)$ expliquent que les membres de la communauté sont à la fois des initiateurs (ils mettent en évidence les problèmes à résoudre ou les améliorations à effectuer), des coproducteurs (ils participent effectivement à la résolution des problèmes) et, enfin, des utilisateurs. D’après les auteurs, cette caractéristique fondamentale du monde de l'open source favorise une création de valeur collective qui est librement partagée. Cela implique un fonctionnement qui dépasse les logiques marchandes, selon la logique du don/contre-don (Alter, 2002). En d'autres termes, les acteurs d'une communauté open source participent selon un principe d'échange (de compétences techniques, de réputation, d'informations, etc.). Lorsqu'un membre de la communauté donne à la communauté, ce dernier sait qu'il ne bénéficiera pas d’une compensation immédiate à sa contribution, mais d’une « compensation différée », dont la nature n'est pas

2 Par souci de simplification, les termes open source et « libre » seront utilisés sans distinction dans la suite du développement. 
connue au moment de l'échange. Or ce sont ces pratiques qui prévalent dans les tiers-lieux, la plupart ayant été fondée par et pour des informaticiens. L'étude des net-up mobilisera nécessairement ces concepts de partage, de don et contre-don et de liberté d'accès des contributeurs.

Notre objectif de recherche visant à comprendre le développement d'un projet entrepreneurial basé sur des dynamiques communautaires au sein d'un tiers-lieu mobilise les pratiques d'analyse de la cocréation, et les différents types de communautés à l'œuvre dans la cocréation. La partie suivante va synthétiser ces facteurs dans une grille d'analyse.

\subsection{Objectifs de la recherche et grille d'analyse pour caractériser la net-up}

Ce travail s'intéresse à un modèle d'entreprise, développé dans un environnement spécifique composé de copyleft, de communautés... et qualifié d'émergent : la net-up. Une net-up est un projet qui se crée et se développe (Burret, 2012) :

- au sein d'un tiers-lieu ;

- autour, par et avec une communauté ;

- par des pratiques de cocréation.

L’objectif général de cette étude consiste, dans une logique exploratoire, à approfondir les concepts-clés à la compréhension de la net-up. Pour réduire le champ de la recherche, la caractéristique «tiers-lieu » est ici considérée comme admise (nous n’étudierons que des cas de projets entrepreneuriaux développés dans des tiers-lieux). Ainsi, la caractéristique « communauté » sera entendue comme l'ensemble des individus regroupés au sein d'un tiers-lieu. Nous souhaitons préciser la nature de la net-up pour comprendre si elle peut être envisagée comme une nouvelle forme du projet entrepreneurial. Ce projet implique de formuler plus précisément notre problématique sous forme des deux questions de recherche suivantes:

Question 1 : comment qualifier la communauté d'une net-up ? Dans les tiers-lieux, les projets entrepreneuriaux se construisent-ils par, autour et avec une communauté qui s'apparente à une communauté de pratique ? Dans le cas d'un tel projet, la communauté en question est déjà préexistante. Dès lors, comment se constitue et évolue la communauté d'une net-up ? L’approche par les communautés caractéristiques du modèle de l'open source proposée dans le cadre théorique offre une piste de solution pour caractériser précisément cette forme communautaire.

Question 2 : par quels mécanismes s'instaure une dynamique cocréative entre un porteur de projet et une communauté présente dans un tiers-lieu ? Il s'agit de s'intéresser au phénomène de cocréation à l'œuvre dans les projets qui se créent et se développent par, autour et avec une communauté et plus précisément, sur les processus d'interaction existants entre le porteur de projet et la communauté que nécessite la cocréation. Il semble que la dimension libre, mise en évidence par certaines pratiques, peut constituer un préalable à la cocréation dans une net-up. 
Pour répondre à ces deux questions de recherche, nous proposons d'analyser les formes de communautés selon les travaux de Wenger (1998) et la dynamique cocréative selon la méthodologie du DATR (Dialogue, Accès, Transparence, Risques).

Wenger (1998) rappelle que les trois critères d'une communauté de pratique sont les suivants :

- l'engagement mutuel : l'appartenance des individus à une communauté de pratique résulte de leur engagement dans des actions qui sont négociées de façon collective. L'engagement mutuel est à l'origine de la cohérence du groupe, que la pratique sert à entretenir. Il est basé sur la complémentarité des compétences et la capacité des individus à transmettre leurs connaissances ;

- l'entreprise commune: les pratiques sociales de la communauté visent à la réalisation d'une entreprise commune (entendu au sens de l'action d'entreprendre). Elle est le « résultat d'un processus collectif permanent de négociation "(Chanal, 2000, p. 8);

- le répertoire commun : est la production de ressources collectives née de la pratique. Il sert de support pour la négociation de sens entre les membres de la communauté. Il peut s'agir d'un ensemble d'informations tangibles (des documents, des outils, des procédures, etc.) ou intangibles (un langage, un raisonnement, etc.). Quant à la communauté épistémique, c'est une communauté qui centre sa pratique sur la production de connaissances et l'apprentissage (Benkeltoum, 2009).

Concernant la cocréation, la méthodologie appliquée dans cet article s’appuie sur les travaux de Prahalad et Ramaswamy (2004) et de Leroy (2008). Afin d'appréhender la mise en place d'une démarche de cocréation avec une communauté, Leroy étudie les interactions entre une entreprise et une communauté de consommateurs, au regard des critères de contrôle du processus de cocréation formalisés par Prahalad et Ramaswamy (2004). Les principes fondamentaux énoncés pour évaluer un processus de cocréation de valeur s'appuient sur le DATR. Le Dialogue, l’Accès, la Transparence et le rapport Risques/Bénéfices constituent les « quatre pavés » d'interactions à étudier. Le Dialogue doit impliquer un réel engagement de la part de l'entreprise et de ses partenaires. Pour maintenir un dialogue qui soit égal, il est indispensable d'assurer l'accès et la transparence des informations. L'Accès à une information passe par une ouverture sur les connaissances et les outils mobilisés par les acteurs engagés. La Transparence de l'information est principalement du ressort de l'entreprise. Ces trois pavés doivent conduire à une évaluation claire du partenaire dans son rapport Risques/ Bénéfices pour l'ensemble de ses actions ou décisions prises lorsqu'il s'engage dans une expérience de cocréation. Selon Leroy (2008), ces quatre pavés peuvent être utilisés en tant que modèle permettant d'analyser les processus de cocréation. Notre grille d'analyse pour caractériser la net-up sera donc la suivante : 
TABleAu 1. GRILle D'ANAlySE POUR CARACTÉRISER LA NET-UP

\begin{tabular}{llll}
\hline Variables étudiées & $\begin{array}{l}\text { Communauté de } \\
\text { pratique }\end{array}$ & $\begin{array}{l}\text { Communauté } \\
\text { épistémique }\end{array}$ & $\begin{array}{l}\text { Cocréation avec une } \\
\text { communauté }\end{array}$ \\
\hline & - Engagement mutuel & - Création de & - DATR: \\
Critères d'analyse & - Entreprise & connaissances & - Dialogue \\
retenus & commune & - Apprentissage & - Accès \\
& - Répertoire commun & & - Transparence \\
& & & - Risques/ \\
& & Bénéfices \\
\hline
\end{tabular}

\section{MÉTHODOLOGIE ET CAS ÉTUDIÉS}

\subsection{Méthodologie : collecte et analyse des données}

Notre recherche, de nature exploratoire, cherche à " définir de nouvelles pistes de recherche, choisir des avenues théoriques ou identifier une méthode appropriée à l'objet " (Trudel, Simard et Vonarx, 2007, p. 42). Une présence active dans plusieurs tiers-lieux en région Rhône-Alpes ${ }^{3}$ (par exemple en prenant part à la vie du lieu, tel que l'accueil du public, la participation et l'organisation des événements, etc.) a permis une intégration totale à la communauté et donc au terrain de recherche. Les deux projets étudiés ont donc effectivement été créés et se sont développés dans un tiers-lieu. Notre stratégie de recherche se rapproche ainsi de la démarche ethnographique visant à produire une description fouillée des terrains étudiés. Selon Rouleau (2013, p. 28), " le but de ces descriptions consiste à mettre au jour les savoirs locaux structurant les liens sociaux et culturels d'un groupe, d'une entreprise ou d'une société ». Cette intégration très tôt dans le projet a permis d'observer l'arrivée de nouveaux membres et de contribuer directement aux interactions. Dans ce contexte, nos deux projets ont été retenus pour servir de cas d'étude, en raison de leur caractère emblématique de projets collectifs menés dans un tiers-lieu, et parce qu'ils permettaient une forme d'observation participante.

Pour permettre l'analyse de contenu par la grille exposée dans la section précédente, le traitement des données s'est focalisé sur la description et l'analyse des deux cas d'étude. Dans un premier temps, une retranscription sous forme de monographies a été effectuée à partir des notes rédigées pendant et majoritairement après la période d'observation. Les deux chercheurs ont repéré les mots-clés associés à la grille d’analyse de manière indépendante, puis ont confronté leur travail afin de valider ces mots-clés. Sur la base de ce travail, ils ont dans un second temps réalisé l'analyse des cas retranscrits en monographies. Les résultats présentés ci-après mettent en lumière les similitudes dans les données observées pour les deux cas d'étude. Pour mettre en évidence certains points de l'analyse, des extraits sont tirés

3 Il s'agit notamment ici du fablab OpenFactory à Saint-Étienne et du bio-hackerspace La Myne à Lyon. 
de ces deux monographies et sont présentés sous la forme d'encadrés. Des verbatim viennent également illustrer certains points clés de la synthèse effectuée.

Plus spécifiquement, concernant le premier cas d'étude - projet Pluggy - la recherche s'est échelonnée sur une période de neuf mois, à raison d'une rencontre de travail toutes les semaines environ. Cette période correspondant à l'entrée du porteur de projet dans la communauté du tiers-lieu jusqu'au développement de son projet par cette même communauté. La collecte des données s'est majoritairement effectuée par le biais d'une observation participante. Deux contenus ont également servi de support matériel. Le premier est un document confidentiel rédigé par le porteur de projet dans le cadre de ses études, présentant à la fois le projet ainsi que la démarche entrepreneuriale. Le deuxième correspond à un support vidéo d'une présentation orale du projet par l'étudiant, filmée lors d'un atelier.

S'agissant du deuxième cas d'étude - projet Mosquito Trap -, le recueil des données est issu de trois sources qualitatives. La première est une observation non participante pendant la période de développement du projet d'une durée de quatre mois. Elle renvoie également à la période d'entrée dans la communauté du tiers-lieu par le porteur de projet, jusqu'à une première phase de développement du projet. La deuxième source s'appuie sur une analyse de contenu décrivant les différentes étapes de développement du projet, rédigé sur la plateforme Movilab. La troisième correspond à un entretien semi-dirigé de 30 minutes mené avec l'un des contributeurs au projet.

\subsection{Cas d'étude}

\subsubsection{Le projet Pluggy}

Pluggy est initialement un projet entrepreneurial porté par un étudiant dans le cadre de ses études. L'idée est née du constat de la difficulté de transférer des données numériques imposantes à une tierce personne, lorsqu'il n'y a pas la possibilité d'utiliser un ordinateur ou Internet. Après un état de l'art des solutions techniques existantes, le porteur de projet s'oriente vers le développement technique d'un dispositif permettant le partage de données numériques de façon hors-ligne, c'est-à-dire sans ordinateur ou connexion internet à disposition. Avec l'aide d'un ami designer et du fablab mis à disposition dans son école, l'étudiant fabrique une maquette non fonctionnelle de démonstration. Dans le but d'accélérer le développement de son projet, tant sur le plan conceptuel que technique, l'étudiant va confronter son projet lors d'un événement organisé par le fablab de Saint-Étienne. Cet événement consiste en un format d'animation, dont l'objectif est de construire un modèle d'affaire innovant, en intégrant les règles de l'open source. L'enjeu de ce format d'animation est de faire connaître auprès des entrepreneurs, les pratiques de l'innovation ouverte et collaborative. Pendant deux jours, le porteur de projet a bénéficié de la collaboration de plusieurs personnes sur son projet, en apportant des compétences et des expertises hétérogènes. C'est à cette occasion que débute la période d'observation. L'étudiant, ayant toujours été intéressé par le monde de l'informatique, a déjà entendu parler de l'open source. Au fil des discussions, il intègre les implications stratégiques qu'induit un développement entrepreneurial basé sur des logiques libres. Après de nombreuses questions posées aux membres de la communau- 
té afin de mieux appréhender ces pratiques, l'étudiant est convaincu du modèle de l'open source pour son projet. À la fin de l'événement, un accord tacite est conclu entre le porteur de projet et plusieurs membres du fablab stéphanois. Sous la condition de placer l'ensemble des contributions faites par les membres du fablab sous licence libre, trois personnes sont intéressées à travailler régulièrement sur le projet. L'étudiant n’ayant pas de connaissances en informatique, deux personnes s'occupent du développement du prototype : un expert en microélectronique, ainsi qu'une étudiante intéressée à se former sur la partie "matériel informatique ». Une troisième étudiante s'engage à soutenir le porteur de projet dans la modélisation et la rédaction du plan d'affaires. Enfin, l'ensemble de la communauté souhaite soutenir le projet en testant régulièrement le prototype, en proposant des améliorations et ainsi devenir le premier marché-test du produit.

\subsubsection{LE PROJET Mosquito Trap}

Mosquito Trap est un projet porté par un professionnel, salarié dans une grande entreprise pharmaceutique. Dans le cadre de son travail, le porteur de projet s'intéresse particulièrement à la technologie dite de la "photo-oxydation » qui, couplée à un dispositif, permet d'assainir l'air ambiant tout en attirant les moustiques dans un piège. Cette technique a été envisagée pour être déployée dans les pays du sud souffrant de maladies propagées par les moustiques. Ce procédé a été testé et validé par le laboratoire pharmaceutique dans lequel travaille le porteur de projet, mais il n’a pas rencontré son marché pour trois raisons : le coût important des appareils existants ; l'absence locale de réseau de maintenance ; l'absence d'un procédé conçu pour être fabriqué et distribué localement. La problématique entrepreneuriale de ce salarié est donc la suivante : « comment mettre cette technologie à la portée du plus grand nombre dans les pays émergents où deux milliards de personnes sont exposées aux maladies portées par les moustiques?» Le projet Mosquito Trap consiste donc à s’approprier cette technologie pour prototyper un appareil qui puisse être fabriqué localement, avec les savoir-faire et les matériaux qui peuvent être disponibles sur place. Grâce à son réseau, l'entrepreneur rencontre une personne qui lui conseille d'aller parler de son projet au fablab de Saint-Étienne. Là, on lui explique que la logique des licences libres pourrait lui permettre de transférer facilement et rapidement sa technologie en s'appuyant sur le réseau des fablabs en Afrique. Par ce biais, la problématique de l'absence de distributeurs dans certains territoires pourrait être contournée. Comme les plans de la mise en œuvre du procédé sont libres, la fabrication peut être prise en charge par un fablab africain, pour être ensuite testée et mise directement en pratique au sein des populations concernées. Après quelques semaines, le fondateur d'un bio-hackerspace à Lyon propose au porteur de projet d'organiser un événement dédié au prototypage du piège à moustique. À cette occasion, quinze personnes aux compétences variées ont travaillé pendant un week-end et trois prototypes ont été réalisés. Le projet est alors communiqué via les concierges jusqu'au fablab d'Abidjan, dont les membres vont développer un prototype avec du matériel local de récupération.

\section{PRÉSENTATION ET ANALYSE DES RÉSULTATS}

Dans les cas d'étude, deux étapes distinctes ont été observées lors du développement de chacun des projets. La première étape correspond à l'arrivée des porteurs de projet dans un 
nouvel environnement (par le biais d'un événement dans le cas de Pluggy, par une visite inopinée dans le cas de Mosquito Trap). Cette « phase d'apprentissage » correspond au moment de l'accueil du porteur de projet par la communauté du tiers-lieu et leurs premières interactions. La deuxième étape, appelée « phase de travail en commun » correspond au moment où la communauté s'engage plus en avant dans un travail effectif sur le projet étudié. Nous distinguerons donc nos autres résultats selon ces deux phases qui constituent notre premier résultat.

\section{1. Étude de la forme communautaire}

Pour rappel, nous souhaitions savoir si la communauté formée autour des projets qui se créent et se développent dans les tiers-lieux, correspond ou non aux caractéristiques des communautés de pratique ou épistémique. En d'autres termes, la cocréation provient-elle de la typologie même de la communauté ? Pour cela, il a été question au travers de l'analyse des données de rechercher les critères énoncés par Wenger (1998) pour chacun des deux cas d'étude.

\subsubsection{Phase D'A PPRentissage}

Les projets choisis comme cas d'étude ont débuté au sein d'un même tiers-lieu : le fablab de Saint-Étienne. L'objectif affiché de la communauté d'un fablab est connu : il consiste à encourager les entrepreneurs à adopter des pratiques empruntées au monde libre dans le développement de leurs projets. La communauté est tournée vers un mécanisme d'apprentissage qui passe par la pratique. Afin d'atteindre cette «entreprise commune », à savoir expérimenter de nouvelles logiques entrepreneuriales, il est nécessaire de construire un "répertoire commun " de ressources, construit de façon tacite par l'échange et le partage d'expérience. Une facette du répertoire commun n'est pas formellement explicitée, mais elle transparaît au travers des conseils et de l'entraide entre les membres (un langage, un jargon, une façon de penser). L'autre facette est plus explicite, dans le sens où ce répertoire commun prend forme sur un support matériel. En effet, le partage des expériences personnelles individuelles et collectives ainsi que les bonnes pratiques sont documentés sur un wiki. Autrement dit, le résultat de ce processus collectif, soit l'entreprise commune, consiste à partager les «bonnes pratiques » éprouvées dans le temps par les membres de la communauté par la documentation. La documentation consiste à décrire les étapes du développement d'un projet, la description des technologies utilisées, le dépôt de code source sur une plateforme, la description des différentes directions que peut prendre le projet, les problèmes à résoudre et les compétences recherchées. Documenter son projet s'apprend. Le porteur de projet doit s'approprier le « langage » du wiki, langage qui n'est pas forcément intuitif : comment créer une page, la modifier, insérer du texte, une photo. Les membres de la communauté sont présents pour aider le porteur de projet à s'approprier ces outils.

On observe que la conscience par les membres de l'identité en tant que groupe est construite à travers les expériences de chacun au sein des activités menées dans le cadre des projets individuels. De par leur pratique, ils enrichissent la connaissance collective. Dans cette communauté, la connaissance est donc apparentée à du « savoir-faire ». Ces caractéristiques 
conduisent à engendrer un "engagement mutuel », formalisé par le soutien et l'entraide entre les membres de la communauté. Ils ne sont pas unis par des liens hiérarchiques, mais par une responsabilité partagée entre chacun des membres. Une responsabilité des uns aux autres orientée vers la réussite de chacun des projets menés au sein de la communauté. Par exemple, dans les deux cas d'études, les projets ont été « accélérés » dans le cadre d'ateliers collaboratifs des fablabs mettant à profit les compétences et les connaissances de la communauté au service des projets.

\section{ENCADRÉ 1. CARACTÉRISATION DES ÉVÉNEMENTS}

Dans le cadre du projet Pluggy, l'atelier « $48 \mathrm{~h}$ chrono » est un format d'animation du type start-up week-end, destiné aux porteurs de projets entrepreneuriaux pour travailler pendant deux jours de façon intensive tout en bénéficiant de la collaboration d'individus aux compétences et expertises hétérogènes. Dans le cadre du projet Mosquito Trap, il s'agit d'un format d'animation appelé protohackaton qui est une méthodologie de développement de projet accéléré permettant, le temps d'un week-end, de prototyper une ou plusieurs variantes de solutions à une problématique (technologique ou de service) précise dans un cadre d'objectifs et de contraintes bien définis.

Individuellement, les membres cherchent à développer leurs compétences pratiques par l'expérimentation. Les membres de la communauté apportent leurs compétences et connaissances, mais en développent également de nouvelles par l'expérimentation. Le porteur de projet quant à lui, applique et expérimente les connaissances mises à disposition, sur son propre projet.

"Toutes les lignes de codes du logiciel sont partagées sous licence libre sur un github ${ }^{4}$ et l'on voit déjà le code un peu évoluer, en dehors du cadre restreint de la petite équipe que l'on a formée. » (Porteur de projet Pluggy)

Pendant la première phase d'apprentissage, la communauté réunie autour des deux porteurs de projet répond aux critères définis par Wenger (1998) concernant les communautés de pratique. La communauté du fablab regroupée autour des projets est composée d'individus aux compétences hétérogènes, mais qui sont engagés dans un même processus de création et de développement entrepreneurial. Ils communiquent et s'entraident sur les façons de mener à bien des projets individuels, par le biais de pratiques empruntées aux logiciels libres.

"Le fait que chacun raconte non seulement à l'oral, mais également sur wiki, les difficultés qu'il a rencontrées et les moyens qu'il a mis en ouvre pour les surmonter permet aux autres de ne pas faire les mêmes erreurs et d'avancer plus vite et mieux sur leur propre projet. " (Porteur de projet Mosquito Trap)

\subsubsection{Phase de travail en COMMUN}

À la suite de la phase d'apprentissage, pour chacun des cas d'étude, un petit groupe d'individus se détache de la communauté pour s'engager dans une phase de travail en commun :

4 Cf. glossaire. 
à la suite d'un événement pour le projet Pluggy, à l'occasion d'un événement pour le projet Mosquito Trap. La logique d'entraide et de soutien au porteur de projet reste toujours prégnante, dans le sens où il perdure encore une volonté de partage d'expérience et bonnes pratiques. Cependant, l'objectif commun à cette nouvelle communauté évolue, et se place au-dessus de ses membres. Il s'agit alors pour ces individus de participer à la création de nouvelles connaissances. Par leur travail, ils souhaitent enrichir le «patrimoine informationnel commun $\|^{5}$ constitué par la communauté. Cela peut prendre toutes les formes : la découverte de nouveaux outils de travail, de nouvelles procédures, de nouvelles techniques, tester ou apprendre d'autres technologies. Aussi, ces connaissances nouvellement acquises nourrissent et augmentent le patrimoine commun à l'ensemble de la communauté.

\section{ENCADRÉ 2. Motivations ET FINALitÉ DE LA COMMUNAUTÉ}

À la fin de l'atelier « $48 \mathrm{~h}$ chrono », plusieurs membres de la communauté font part au porteur de
projet de leur envie de s'engager plus en avant dans le développement de son projet. Un accord tacite
est verbalement formalisé. L'étudiant expose simplement ses propres échéances et les objectifs qu'il
souhaite atteindre. Les membres du groupe commencent alors à travailler sur le projet. Pour eux,
les motivations sont multiples. Pour la personne s'occupant de la programmation informatique, ses
motivations sont bénévoles, elle souhaite simplement apporter ses compétences. Pour l'étudiante
en charge de l'assemblage du matériel informatique, il s'agit d'apprendre de nouvelles compétences
sous la supervision du programmeur. Pour la deuxième étudiante, sa motivation est de mettre en
pratique ses compétences et de professionnaliser sa démarche. Pour tous, au-delà des motivations
personnelles, un objectif leur est commun : créer de nouvelles connaissances. Adhérents à la
communauté du fablab, ils connaissent les pratiques et la philosophie du lieu. La finalité est une
production de connaissance qui soit libre et ouverte. Projet Pluggy

En participant collectivement à la construction du patrimoine commun de connaissances, les initiatives individuelles pourront en bénéficier. Chacun des membres de la communauté pourra s'appuyer sur ces nouvelles connaissances pour développer ses propres projets.

" Je laisse les plans du projet sous licence libre. Je n'ai pas le temps de le prototyper, du moins, pas tout de suite. Par contre, la petite équipe du hackerspace l'a déjà bien fait évoluer et je crois bien qu'un fablab en Côte-d'Ivoire travaille en ce moment sur un premier prototype. " (Porteur du projet Mosquito Trap)

Parfois, c'est même une nouvelle communauté qui se mobilise autour du projet.

5 On entend par patrimoine informationnel commun l'ensemble des savoirs, des pratiques, des actions, etc. produits au sein d'un tiers-lieu, et regroupés sous la forme d'une documentation dans un wiki. Voir le Manifeste des tiers-lieux pour aller plus en avant sur cette notion. 
Les membres du bio-hackerspace de Lyon prennent en charge l'organisation de l'événement. Ils s'occupent de trouver un lieu, de rassembler des matériaux de récupération et de créer et diffuser les supports de communication. Ils effectuent un appel à participation auprès de leurs communautés respectives. Pendant l'événement, ils se chargent également de l'animation des équipes formées, de la restauration, ainsi que de la documentation du déroulé du hackaton. Quinze personnes répondent à l'invitation et participent bénévolement à l'événement. Ils sont de profils très diversifiés : scientifique, étudiant, graphiste, etc. Pendant tout un week-end, ces personnes travaillent en équipe de cinq au prototypage d'une solution basée sur le code source initial du projet. Il est intéressant de noter qu'à partir de cet événement, l'identité du porteur de projet initial s'efface au profit du projet en lui-même. Le salarié s'est détaché de l'événement, ne participant pas à son organisation ou à son déroulement. Après l'événement, le projet s'est vu réapproprié par quelques-uns des participants. Une nouvelle communauté s'est formée autour du projet, et non plus autour du porteur de projet. Mosquito Trap continue son développement au sein d'une autre communauté composée d'autres personnes extérieures au fablab de Saint-Étienne, sur les premiers acquis documentés par le porteur de projet et les membres du fablab stéphanois. Projet Mosquito Trap

Ces communautés nouvellement créées sous l'impulsion d'un projet semblent s'apparenter au modèle de la communauté épistémique (la pratique est sous-entendue comme le prétexte de l'apprentissage). On comprend que les communautés activées dans les créations de net-up sont différentes selon les phases du projet : communauté de pratique dans la phase d'apprentissage puis un mélange de communauté de pratique et de communauté épistémique ensuite.

\section{2. Étude de la démarche de cocréation avec une communauté}

À partir des cas étudiés, la grille de lecture fournie par les critères du DATR montre une évolution des interactions entre la communauté et le porteur de projet. En effet, les critères réagissent différemment selon la phase d'apprentissage ou la phase de travail en commun. Dans la première phase, le porteur de projet intègre un tiers-lieu, mais également la communauté du tiers-lieu. Un premier dialogue se met en place, destiné à transmettre un ensemble d'informations permettant au porteur de projet de décider de s'engager ou non dans la dynamique communautaire. Ce dialogue s'oriente principalement de la communauté vers le porteur de projet. Lorsque l'objectif d'un dialogue «égal » est atteint, le porteur de projet peut se sentir suffisamment en confiance pour partager de façon plus précise son idée avec les membres de la communauté.

\section{ENCADRÉ 4. INTÉGRATION DU PORTEUR DE PROJET DANS UNE COMMUNAUTÉ}

Le porteur du projet Pluggy a pu immédiatement s'immerger dans l'atmosphère et l'ambiance propres au travail collaboratif dans un tiers-lieu, grâce à sa participation à un événement dédié (« $48 \mathrm{~h}$ chrono »). À cette occasion, plusieurs ateliers pratiques sont aménagés : l'atelier de prototypage, où 
sont installés plusieurs imprimantes 3D, des bobines de fil plastiques, des prototypes exposés sur les tables ; l'atelier informatique où du matériel informatique de récupération est entassé sur plusieurs tables ; et enfin, des tables avec des crayons de couleur, des feutres, des post-it ainsi que plusieurs paperboards forment l'atelier sur la construction de business model. Pour cet événement, une cinquantaine de personnes est mobilisée pour travailler sur les projets, en apportant conseils et compétences. Ils sont pour la plupart entrepreneurs, mais il y a également des étudiants, des salariés, des curieux. Plusieurs compétences sont représentées : design, développement informatique, comptabilité, etc.

Dans le cas du projet Mosquito Trap, le salarié rencontre la personne chargée au sein d'un tiers-lieu d'accueillir les nouveaux arrivants et d'animer la communauté : le concierge. Le porteur de projet ayant vaguement entendu parler des fablabs et autres hackerspaces, le concierge lui explique leur fonctionnement, leur éthique et leur philosophie. À son tour, le porteur de projet lui raconte son parcours professionnel et lui expose le projet « Mosquito Trap». Le porteur de projet prend l'habitude de venir tous les lundis, afin de rencontrer les adhérents du fablab. Les discussions sont pour la plupart tournées vers les différentes manières de récupérer et de transformer du matériel. Informés par le concierge du projet en cours à Saint-Étienne, les membres du fablab d'Abidjan commencent de leur côté à rassembler du matériel de récupération en vue d'un futur prototypage du projet.

Le phénomène de la cocréation dans la première phase est difficile à appréhender dans le sens où elle s'exprime au travers des différentes interactions entre la communauté et le porteur de projet. La communauté aide et soutien le porteur de projet à modéliser son projet grâce à des logiques entrepreneuriales inspirées des logiques libres. Les membres proposent de nouvelles idées, conseillent des outils, des pratiques, partagent leur expérience. C'est par ces différentes interactions que le porteur de projet adopte de nouveaux mécanismes, notamment en prenant en compte la nécessité de fédérer une communauté autour de son projet. Dans ce contexte, la cocréation n'est de premier abord pas visible de façon concrète sur le produit ou sur le service, mais plutôt dans la manière de le développer : le choix de la technologie utilisée, le modèle d'affaires, le mode de financement, les usages, etc.

Pendant cette phase d'apprentissage, l'accès et la transparence des informations sont facilités par une contrainte érigée par la communauté et déjà abordée dans la section précédente : la documentation. Divers outils sont mis en place pour faciliter cette pratique, souvent par l'utilisation d'un wiki.

\section{ENCADRÉ 5. LA PRATIQUE DE LA DOCUMENTATION}

Pour initier une dynamique collaborative pour le projet Mosquito Trap, le concierge conseille au porteur de projet de se plier à une étape importante : la documentation de son projet, et en particulier de la solution technique telle qu'elle est à l'état actuel. La documentation est une étape fondamentale et incontournable dans les pratiques du libre. Selon le concierge, elle prouve la volonté de partager, nécessaire à la réappropriation par les tiers. Ce dernier lui ouvre alors un compte sur le wiki dédié du fablab et lui explique les différentes étapes ainsi que la forme que doit prendre la documentation, suivant un formulaire spécifique. 
Pendant la phase d'apprentissage, la documentation semble à la fois encourager et favoriser la collaboration des membres de la communauté (en assurant les critères d'accès et transparence de l'information). Pour la communauté, cette pratique s'apparente à une règle fondamentale. La documentation permet à la communauté d'appréhender le rapport Risques/ Bénéfices à «initier » le porteur de projet par l'entraide et le partage de connaissances. Pour le porteur de projet, elle démontre une volonté de s'engager auprès de la communauté. Elle donne le signal d'un premier partage d'informations, encourageant les autres membres à s'approprier le projet. "La peur de se faire voler son idée prévaut au début, mais il faut bien se rendre compte de deux choses. La première, c'est que je ne suis pas le premier à avoir cette idée. La deuxième est que la communauté open source a des capacités de développement de projet conséquentes. » (Porteur du projet Pluggy)

À la suite de cette phase d'apprentissage, certains membres de la communauté dépassent le stade de l'entraide pour s'engager dans une dynamique collaborative. Ici, le phénomène de cocréation est plus facile à appréhender. Cette deuxième phase répond à la définition de la cocréation dans le sens où un travail commun est mené en vue de trouver des solutions conjointes à une problématique définie en amont par le dialogue entre le porteur de projet et la communauté. Plusieurs compétences sont agrégées pour répondre à la problématique. Parfois le porteur de projet adopte une posture de dirigeant, parfois la communauté s'autoorganise. Les risques, mais également, les bénéfices, sont d'autant plus accentués dans cette phase. Pour le porteur de projet, adopter une logique libre pour son projet revient à prendre le risque de se voir "voler son idée ». Mais les bénéfices consistant à acquérir des compétences sans lesquels le projet ne pourrait pas être développé pallient ce risque.

Pour la communauté, le risque d'engager du temps et des compétences sur un projet est minimisé par la pratique de la documentation. Si l'entrepreneur décide d'arrêter son projet, ou de prendre une direction qui ne correspond plus aux valeurs de la communauté, le travail fourni en commun reste accessible. Le projet peut également se séparer en plusieurs autres projets, qui peuvent suivre des voies différentes, selon la logique du « fork ${ }^{6} »$. Pendant la phase de travail en commun, la documentation systématique s'apparente à une contrepartie requise par les membres de la communauté pour leur apport de compétences dans le développement d'un projet (c'est-à-dire en contribuant à minimiser le rapport Risques/ Bénéfices de la communauté).

Retenons que la documentation systématique de tous les stades du projet favorise une vision étendue de la propriété intellectuelle, qui devient un élément caractéristique de la création d'une net-up.

\section{CONCLUSION}

Au niveau de la caractérisation des communautés impliquées dans les projets de net-up, il est intéressant de retenir que, dans la phase d'apprentissage, la communauté et les porteurs de projet expérimentent de nouvelles logiques entrepreneuriales (apprentissage) en travail-

6 Cf. glossaire. 
lant sur leur propre projet et en participant aux autres projets de la communauté (pratique). L'apprentissage, individuel, est bien le résultat d'une pratique, ce qui caractérise les communautés de pratique. Dans la phase de travail en commun, la communauté se modifie : un petit groupe de membres se détache pour s'engager plus fortement dans le projet, et ouvre la possibilité à d'autres individus intéressés de les rejoindre. Leur volonté est clairement d'enrichir un stock de connaissances commun. Les membres de la communauté s'engagent dans le développement d'un projet (pratique) dans le but de produire de nouvelles connaissances (apprentissage). La pratique devient alors un moyen d'apprendre collectivement, ce qui caractérise les communautés épistémiques. Il devient alors possible de répondre à la première question de recherche. La communauté regroupée autour d'un projet qui se crée et se développe au sein d'un tiers-lieu s'apparente à une forme hybride ${ }^{7}$ entre la communauté de pratique et la communauté épistémique. Cette « hybridation » s'observe lors de l'enclenchement d'une démarche collaborative de travail effectif sur les projets étudiés. En effet, la communauté mobilisée dans un projet de net-up commence par être une communauté de pratique, pilotée par les pratiques des tiers-lieux, puis évolue vers une communauté épistémique au fur et à mesure du développement du projet. Au final, la communauté mobilisée est un mélange de communauté de pratique (qui perdure tout au long du projet), et de communauté épistémique qui apparaît spontanément lorsque le projet prend de l'ampleur. Parfois même, cette communauté hybride finit par remplacer le porteur de projet dans la création de la net-up (cas Mosquito Trap).

Pour répondre à la deuxième question de recherche, une gestion étendue de la propriété intellectuelle (observée dans des pratiques empruntées au monde du libre), caractérisée, entre autres, par la documentation systématique des avancées du projet, favorise la cocréation dans un projet qui se crée et se développe par, autour et avec une communauté. Le tableau 2 propose de regrouper l'ensemble des points clés tirés de l'analyse des résultats, en croisant les différentes phases observées et les formes de communautés impliquées, avec les critères de contrôle de la cocréation du DATR.

7 Pour reprendre les termes utilisés par Cohendet, Creplet et Dupouët (2003). 
TABLEAU 2. SynthèSE DE L'ANALYSE DES RÉSUltatS POUR UNE CARACTÉRISATION DE LA NET-UP

\begin{tabular}{|c|c|c|c|}
\hline $\begin{array}{ll} & \text { DATR } \\
\text { Phases } & \end{array}$ & Dialogue & Accès/Transparence & Risques/Bénéfices \\
\hline $\begin{array}{l}\text { Phase } \\
\text { d'apprentissage }\end{array}$ & $\begin{array}{l}\text { Le dialogue entre le } \\
\text { porteur de projet et la } \\
\text { communauté de pratique } \\
\text { s'axe sur l'apprentissage } \\
\text { des meilleures } \\
\text { pratiques. Les membres } \\
\text { cherchent à améliorer } \\
\text { leurs compétences } \\
\text { individuelles par } \\
\text { l'entraide. }\end{array}$ & $\begin{array}{l}\text { L'accès et la } \\
\text { transparence des } \\
\text { informations s'effectuent } \\
\text { par la transmission d'un } \\
\text { répertoire commun } \\
\text { de ressources. Cette } \\
\text { transmission s'effectue } \\
\text { de façon tacite (par } \\
\text { les échanges avec } \\
\text { la communauté) } \\
\text { ou explicite (par la } \\
\text { documentation des } \\
\text { «bonnes pratiques », } \\
\text { dont le contenu est } \\
\text { accessible sur une } \\
\text { plateforme collaborative } \\
\text { dédiée). }\end{array}$ & $\begin{array}{l}\text { Le dialogue permet à } \\
\text { chacune des parties } \\
\text { d'appréhender leur } \\
\text { rapport Risques/ } \\
\text { Bénéfices. Pour le } \\
\text { porteur de projet, } \\
\text { il s'agit de disposer } \\
\text { d'un maximum } \\
\text { d'informations sur } \\
\text { les avantages et les } \\
\text { contraintes pour intégrer } \\
\text { la communauté. }\end{array}$ \\
\hline $\begin{array}{l}\text { Phase de travail } \\
\text { en commun }\end{array}$ & $\begin{array}{l}\text { Le dialogue entre } \\
\text { le porteur de projet } \\
\text { et la communauté } \\
\text { épistémique est orienté } \\
\text { sur la production } \\
\text { de nouvelles } \\
\text { connaissances. Les } \\
\text { membres cherchent à } \\
\text { alimenter le patrimoine } \\
\text { informationnel commun } \\
\text { de la communauté. }\end{array}$ & $\begin{array}{l}\text { L'accès et la } \\
\text { transparence des } \\
\text { informations deviennent } \\
\text { l'élément central } \\
\text { de la collaboration. } \\
\text { La documentation } \\
\text { semble faire office } \\
\text { de contrepartie, en } \\
\text { « échange » des } \\
\text { compétences et des } \\
\text { ressources apportées } \\
\text { par les membres de la } \\
\text { communauté. }\end{array}$ & $\begin{array}{l}\text { La contrepartie offerte } \\
\text { par la documentation du } \\
\text { projet minimise le risque } \\
\text { pour la communauté } \\
\text { de fournir du travail } \\
\text { sans rétribution. Le } \\
\text { risque pour le porteur } \\
\text { de projet de se «faire } \\
\text { voler son idée» est } \\
\text { contrebalancé par le } \\
\text { fait d'acquérir des } \\
\text { compétences qu'il ne } \\
\text { pourrait trouver ailleurs } \\
\text { sans contrepartie } \\
\text { financière. }\end{array}$ \\
\hline
\end{tabular}

Cette synthèse met en évidence des éléments qui complètent la littérature sur l'analyse de la cocréation et les premiers éléments de définition des net-up. Elle montre qu’on peut étendre l'analyse DATR à d'autres formes de communautés que les clients, et qu'il peut être pertinent de différencier des phases dans le projet de cocréation analysé. Nous constatons ainsi que le Dialogue ne s'oriente pas vers les mêmes objectifs en fonction de la phase du projet, et qu'il peut jouer un rôle important dans la mesure de l'équilibre Risques/Bénéfices. Il est également au centre de la construction d'un patrimoine commun quand le projet de net-up se précise et est pris en charge par une communauté épistémique. L'Accès et la Transparence sont ren- 
dus possibles par des pratiques à la fois tacites et explicites. Ces deux critères de contrôle deviennent des piliers de la cocréation dans un esprit open source, car ils garantissent la logique de don/contre-don qui lui est associée. Quant à l'équilibre Risques/Bénéfices, il est garanti, pour les deux parties, par la documentation systématique imposée par la logique open source du tiers-lieu.

Les réponses apportées aux deux questions de recherche grâce aux résultats présentés cidessus nous permettent finalement de proposer une " extension » de la définition de la netup (Burret, 2012). Dès lors, une net-up est un projet qui se crée et se développe :

- au sein d'un tiers-lieu ;

- autour, par et avec une communauté, qui s'apparente à une forme hybride entre la communauté de pratique et la communauté épistémique ;

- par des pratiques de cocréation, qui sont permises par une gestion étendue de la propriété intellectuelle (systématisation d'une documentation notamment).

Les tiers-lieux impliqués dans notre étude sont des espaces très collectifs, parfaitement en cohérence avec le Manifeste des tiers-lieux, qui prônent le partage et la documentation systématique des projets dans la logique open source informatique. Mais les espaces collaboratifs ne partagent pas tous les mêmes valeurs et les mêmes objectifs (Bottollier-Dubois et al., 2014 ; Germain, 2017). Certains par exemple ne respectent pas la charte des tiers-lieux, et ne poussent donc pas aussi fortement les valeurs du libre que les tiers-lieux de notre étude. De plus, nous pouvons distinguer les espaces collaboratifs dans lesquels le temps imparti au porteur de projet est limité, ce qui applique une certaine pression à la réussite; et les espaces collaboratifs plus communautaires, caractérisés par une non-maîtrise de la productivité et où le résultat obtenu est très souvent une conséquence non intentionnelle du travail de l'individu porteur de projet. Cette différenciation est importante à prendre en considération lorsqu'on s'intéresse aux net-up, et plus largement aux nouvelles formes d'entrepreneuriat développées dans des lieux collaboratifs, car le temps et la volonté de réussite individuelle ou collective sont les deux éléments clés de ce type de projets. Si la documentation systématique n’est pas imposée, il existe des risques élevés que les acteurs en présence n’aient pas confiance les uns dans les autres aussi rapidement et clairement que dans nos deux cas, ce qui hypothèque évidemment les chances de réussite de la net-up. Il serait souhaitable de renouveler l'observation en affinant la variable tiers-lieux en fonction d'une typologie plus détaillée (De Vaujany, Bohas, Fabbri et Laniray, 2016) : espaces de coworking, makerspaces, fablabs, hackerspaces, incubateurs, accélérateurs, digital labs...

Les tiers-lieux de notre étude se caractérisent également par le fait qu'ils sont des espaces de communs; des endroits où les membres de la communauté et les porteurs de projet se rejoignent dans une vision commune de création de valeur basée sur des notions d'usages et de services, de dons et contre-dons. Ces espaces font de la création de communs une priorité (Ostrom, 2010 ; Paranque, 2014) ; l'objectif est de partager de la connaissance et des pratiques. La connaissance est un commun particulier qui croît en étant partagée : plus elle est mobilisée plus elle se développe. Cela est rendu d'autant plus aisé que les technologies de l'information et de la communication actuelles et très présentes dans les tiers-lieux rendent le coût de ce partage quasi nul (Rifkin, 2014). Selon Coriat (2015), un commun renvoie à trois séries de déterminants : la « ressource » mise en commun et partagée (dans nos cas une 
maquette non fonctionnelle et la technologie de "photo-oxydation »), le mode d'accès à cette ressource et les règles de son partage (l'obligation de partage et de documentation systématique par tous les acteurs du projet), enfin le mode de gouvernance de la ressource (pilotée par les types de communautés qui se fédèrent autour du projet). Dans cette problématique le mode d'organisation et l'action collective sont hautement stratégiques... pas de commun sans commoners. Dès lors, le modèle dessiné par la net-up semble proposer une nouvelle forme entrepreneuriale, car elle dépasse une simple logique de capitalisation et de concurrence pour aller vers une production de bien commun, dans un modèle de développement distribué. L'objectif étant pour le porteur de projet de créer une entreprise lucrative et/ou de donner la possibilité à d'autres membres de la communauté d'en créer une en exploitant autrement la connaissance créée collectivement (Suire, 2016). En somme, une vision entrepreneuriale qui n'est pas déconnectée d'une création de valeur «monétarisée », mais qui est couplée à une création de valeur "sociétale ( Beraud et Cormerais, 2011). Les dynamiques de création collective observées dans les net-up pourraient alors s'envisager sous l'angle de la contribution. Dans ce cas, il ne s'agirait plus d'évaluer seulement les stratégies de cocréation, mais aussi d'en évaluer les logiques de contribution/rétribution : le porteur de projet bénéficie-t-il plus que la communauté du commun ou est-ce l'inverse ? Pour le cas Pluggy, le porteur de projet a probablement tiré plus de profit de la cocréation que le fablab. Par contre, le porteur du projet Mosquito Trap a fini par céder complètement la technologie qu'il apportait au profit de la communauté qu'il avait fédérée, et plus encore au profit d'une communauté à l'étranger avec qui il n'avait pas eu de contact direct. Nous voyons là l'imbrication intéressante d'un espace de communs (un fablab) avec un projet porté par les communautés de cet espace, car porteur de futurs communs (les fondements et développements, documentés, de la net-up). Ce résultat émergent s'inscrit dans les cas concrets illustrant ce nouveau paradigme économique et stratégique qu'est la création de communs (Morse, 2010 ; Desreumaux et Brechet, 2013 ; Tirole, 2016), avec une vision entrepreneuriale. Il s'agit probablement d'une voie de recherche prometteuse pour les futurs travaux en entrepreneuriat.

Cette approche entrepreneuriale qui consiste à amener son projet dans un espace de communs nous laisse penser qu'il serait pertinent d'analyser nos résultats en adoptant une posture épistémologique pragmatiste de l'action collective (Brechet et Schieb-Bienfait, 2011 ; MacLean, Macintosh et Seidl, 2015) en lien avec la théorie de l'agir créatif (Joas, 2005) et de l'agir projectif (Emirbayer et Mische, 1998). L’agir créatif correspond à la prise en compte de la créativité comme ouverture à de nouvelles façons d'agir, en interaction avec les parties prenantes; l'action n'est donc pas vue comme le résultat d'un calcul rationnel, mais comme encastrée socialement et autoréflexive. Les net-up sont inscrites dans l'agir créatif dans le sens où leurs porteurs de projets s'engagent dans une forme d'improvisation intentionnelle. Les porteurs de projets entrepreneuriaux arrivent dans le tiers-lieu volontairement, mais souvent sans trop savoir quoi en attendre et sans savoir comment les membres de la communauté présente vont réagir au projet qu'ils portent. Quant à l'agir projectif, il mêle les considérations existentielles et opératoires d'un projet si celui-ci est considéré au-delà de sa dimension technique. Dans le cas de la net-up, il y a tout d'abord un façonnement conjoint de l'acteur et du contexte dans une phase d'émergence organisationnelle, qui correspond dans nos cas d'étude à la phase dite d'apprentissage. Nous avons également observé une évolution en parallèle d'un projet individuel et collectif qui correspond à un «engagement social fondé sur une capacité au présent de se nourrir du passé et du futur imaginé pour envisager l'action » 
(Brechet et Schieb-Bienfait, 2011, p. 38). Le cas Mosquito Trap illustre bien cette évolution en parallèle d'une envie individuelle de partage d'une technologie et d'une appropriation collective de cette technologie, et au-delà, de la volonté de diffusion et d'entraide internationales. Le concept de "néotribu » de Maffesoli (1988) nous semble illustrer au mieux cette envie des jeunes (ou moins jeunes !) entrepreneurs à réagir aux transformations socioéconomiques post-industrielles en formant des collectifs éphémères liés à des styles de vie et à des intérêts communs. Cette configuration entrepreneuriale créative, aux objectifs à la fois individuel et collectif de création de communs, mérite vraiment, selon nous, la curiosité des chercheurs en entrepreneuriat.

\section{GLOSSAIRE}

Code source : le code source est un texte produit par un humain qui représente les instructions que doit effectuer un microprocesseur pour exécuter un programme. Le code source est écrit dans un langage de programmation qui permet de faire l'interface entre l'homme et la machine.

Coworking : Spinuzzi (2012) définit les espaces de coworking comme des environnements ouverts où des individus non affiliés disposent de conditions de travail en s'acquittant d'un montant mensuel fixe pour exercer une activité professionnelle.

Crowdsourcing : par définition le crowdsourcing est l'externalisation d'une activité (to out source) vers la foule (crowd).

Fablab : un fablab, ou fabrication laboratory (laboratoire de fabrication) est un « espace ouvert dédié à la fabrication d'objet et équipé de machines numériques » (imprimantes 3D, découpeuses laser). Le concept de fablab vient des États-Unis, dans l'université MIT où le premier fablab a été créé. Les fablabs sont un prolongement de la culture libre, dans le sens où ils favorisent la diffusion des savoirs et des pratiques, appliquée ailleurs que sur les logiciels. En ce sens, la charte des fablabs oblige à respecter les fondements du libre à propos de la diffusion du savoir produit au sein des laboratoires de fabrication.

Fork/Forker : traduit en français par « embranchement » ou "fourchette », un fork est un nouveau logiciel créé à partir d'une copie du code source d'un logiciel existant. Forker signifie « créer un projet indépendant à partir d'un projet existant, sans détruire celui-ci » (Zacchiroli, 2014). Cette pratique est autorisée étant donné les principes d'utilisation, de modification et de redistribution du code source autorisé par les licences libres.

Github : github est un des outils de développement collaboratif utilisés par les développeurs de logiciels libres en vue d'améliorer leur efficacité et leur productivité.

Hackerspace/Bio-hackerspace : un hackerspace (ou hacklab) est un "espace physiquement situé où des personnes ayant un intérêt pour les techniques se rassemblent pour travailler sur des projets tout en partageant des équipements (machines-outils, wifi...), des connaissances et des valeurs communes issues de l'éthique des hackers ». Dans le cas où le hackerspace s'intéresse particulièrement aux thématiques portant autour de la biologie et du vivant, il est nommé bio-hackerspace ou éco-hacklab. Un cadre juridique et éthique spécifique est mis en place au sein de ces bio-hackerspaces. Les projets menés y sont exclusivement en open source, 
dans une logique de non-brevetabilité des techniques et des connaissances liées au monde du vivant.

Lead users : Von Hippel (1986) propose une définition du lead user en tant qu'« utilisateur qui éprouve aujourd'hui des besoins forts qui se généraliseront au reste du marché, des mois ou des années après ; il retire un bénéfice significatif de produits qui offrent des solutions répondant à ses besoins insatisfaits par le marché ».

Licence : une licence est un contrat juridique permettant à un tiers d'exploiter un droit de propriété intellectuelle (par exemple une marque ou un brevet), à titre onéreux ou gratuit.

Movilab : au sein de la communauté du fablab de Saint-Étienne, il est d'usage d'effectuer les documentations sur une plateforme wiki dédiée, appelée Movilab. C'est en croisant les logiques du libre et du durable que Movilab a créé une méthodologie visant à expérimenter des solutions opérationnelles. De la même manière que le libre partage les codes source d'un logiciel, Movilab encourage le partage des codes sources (ou " recettes ») des projets. En d'autres termes, de transposer les pratiques issues du monde des logiciels libres et open source, adaptées dans la documentation de projets dits « remarquables » pour leur participation au déploiement des modes de vie durables.

Tiers-lieu : le concept de tiers-lieu peut s'entendre de deux manières : la notion de «tierslieu de travail » (Boboc, Bouchareb, Deruelle et Metzger, 2014) est utilisée pour qualifier les dispositifs de type : espace de coworking, télécentre, etc. permettant aux télétravailleurs et aux travailleurs indépendants d'exercer leurs activités professionnelles dans un environnement matériel approprié autour d'un réseau de sociabilité. Il renvoie également à la notion de «tiers-lieu de fabrication » utilisé dans le langage de spécialité pour qualifier les dispositifs de type : fablabs ou hackerspaces, etc. qui mettent à disposition d'individus divers des ressources techniques et des machines de qualités professionnelles dans un réseau de sociabilité. À ce niveau de compréhension, l'approche à considérer ici rejoint l'analyse de Burret $(2014,2015)$ selon lequel les tiers-lieux représentent un levier favorable pour « la création et la production de nouveaux produits ou services à valeur ajoutée ».

Wiki : selon la définition de Wikipédia, un wiki est une application web qui permet la création, la modification et l'illustration collaboratives de pages à l'intérieur d'un site web. C'est un outil de gestion de contenu, dont la structure implicite est minimale, tandis que la structure explicite émerge en fonction des besoins des usagers.

\section{RÉFÉRENCES}

Alter, N. (2002). Théorie du don et sociologie du monde du travail. Revue du MAUSS, 2(20), 263-285.

Ayerbe, C. et Chanal, V. (2011). Quel management des DPI dans les business models ouverts. Revue française de gestion, 1(210), 99-115.

Bécheur, A. et Gollety, M. (2006). Validation d'une échelle de mesure du lead user. Revue Française du Marketing, 206(1-5), 29-39.

Benkeltoum, N. (2009). Les régimes de l'open source : solidarité, innovation et modèle d'affaires. Business administration. Paris, École nationale supérieure des mines de Paris. 
Beraud, P. et Cormerais, F. (2011). Économie de la contribution et innovation sociétale. Innovations, 1(34), 163-183.

Boboc, A., Bouchareb, K., Deruelle, V. et Metzger, J.-L. (2014). Le coworking: un dispositif pour sortir de l'isolement. SociologieS. Récupéré en ligne le 19 avril 2017 sur le site : http://sociologies.revues. $\operatorname{org} / 4873$

Bottollier-Dubois, F., Dalle, B., Eychenne, F., Jacquelin, A., Kaplan, D., Nelson, J. et Routin, V. (2014). États des lieux et typologie des ateliers de fabrication numérique (rapport au ministère de l'Économie).

Brechet, J.-P. et SChieb-Bienfait, N. (2011). L'entrepreneuriat confronté au pluralisme théorique : la nécessité d'une project-based view. Revue de l'Entrepreneuriat, 2(10), 29-44.

Burger-Helmchen, T. et Pénin, J. (2011). Crowdsourcing : définition, enjeux, typologie. Revue management \& avenir, 1(41), 254-269.

Burret, A. (2012). Au-delà des startups les net-ups. Revue Économique et Sociale, 70(4).

Burret, A. (2014). Étude exploratoire des tiers-lieux comme dispositif d'incubation libre et ouvert de projet. $23^{e}$ Conférence internationale de management stratégique. Rennes, 26-28 mai.

Burret, A. (2015). Tiers lieux et plus si affinités. Limoges, FYP Éditions.

Chanal, V. (2000). Communautés de pratique et management par projet : à propos de l'ouvrage de Wenger (1998) «Communities of practice : learning, meaning and identity ».M@n@gement, 3(1), 1-30.

Chesbrough, H.W. (2003). Open innovation: the new imperative for creating and profiting from technology. Boston, Massachusetts, Harvard Business School Press.

Cohendet, P., Creplet, F. et Dupouët, O. (2003). Innovation organisationnelle, communautés de pratique et communautés épistémiques : le cas de Linux. Revue française de gestion, 29(146), 99-121.

Comtesse, X. et Pauletto, G. (2010). Comment les réseaux sociaux peuvent-ils favoriser la créativité et l'innovation régionales. Revue Économique et Sociale, 68(2), 17-25.

Coniat, B. (2015). Le retour des communs. La crise de l'idéologie propriétaire. Paris, Les Liens qui Libèrent.

De Vaujany, F.X., Bohas, A., Fabbri, J. et Laniray, P. (2016). Nouvelles pratiques de travail : la fin du clivage salariat-entrepreneuriat? Groupe de recherche RGCS.

Desreumaux, A. et Brechet, J.-P. (2013). L'entreprise comme bien commun. Revue Interdisciplinaire Management, Homme \& Entreprise, 3(7), 77-93.

ELLIOTt, M.-S. et SCACCHI, W. (2003). Free software : a case study of software development in a virtual organizational culture. Oakland, Californie, University of California.

Emirbayer, M. et Mische, A. (1998). What is agency. American Journal of Sociology, 103(4), 962-1023.

Germain, O. (2017). Théories en entrepreneuriat : pour que les fruits passent la promesse des fleurs. Dans J. Saint-Pierre et F. Labelle (dir.), Les PME d'hier à demain (p. 17-65). Presses de l'Université du Québec, Canada.

HAMDI-KIDAR, L. (2013). Cocréation marketing de produit avec les consommateurs : Quelle(s) cible(s) choisir? (thèse de doctorat en gestion). Toulouse, Université Toulouse 1 Capitole.

JoAs, H. (2005). The creativity of action. Cambridge, Polity Press. 
Leroy, J. (2008). Gestion de la relation avec une communauté virtuelle dans une stratégie de cocréation. Les leçons du cas Fon.com. Décisions Marketing, 52, 41-50.

Loilier, T. et Tellier, A. (2004). Comment peut-on se faire confiance sans se voir ? Le cas du développement des logiciels libres. M@n@gement, 7(3), 275-306.

Loilier, T. et Tellier, A. (2011). Que faire du modèle de l'innovation ouverte ? Revue française de gestion, 1(210), 69-85.

Maclean, D., Macintosh, R. et Seidl, D. (2015). Rethinking dynamic capabilities from a creative action perspective. Strategic Organization, 13(4), 340-352.

Madanmohan, T.R. et NavelKar, S. (2002). Roles and knowledge management in online technology communities : an ethnography study. Bangalore, Bangalore Indian Institute of Management.

Maffesoli, M. (1998). Société ou communauté. Tribalisme et sentiment d'appartenance. Corps et culture, 3. Récupéré le 12 octobre 2007 du site : http://corpsetculture.revues.org/520.

Morse, R.S. (2010). Integrative public leadership : catalyzing collaboration to create public value. The Leadership Quarterly, 21, 231-245.

Murnieks, C.Y., Cardon, M.S., Sudek, R., White, T. et Brooks, W.T. (2016). Drawn to the fire : the role of passion, tenacity and inspirational leadership in angel investing. Journal of Business Venturing, 31(2016), 468-484.

Ostrom, E. (2010). Gouvernance des biens communs. Bruxelles, De Boeck.

PARAnque, B. (2014). Laction collective coordonnée autour de la gestion de ressources communes. La Revue des Sciences de Gestion, 5(269-270), 75-82.

Prahalad, C.K. et Ramaswamy, V. (2004). Cocreation experiences : the next practice in value creation. Journal of Interactive Marketing, 18(3), 5-14.

Rifkin, J. (2014). La nouvelle société du coût marginal zéro. L'Internet des objets, l'émergence des communaux collaboratifs et l'éclipse du capitalisme. Paris, Babel.

Rouleau, L. (2013). L'ethnographie organisationnelle d'hier à demain. Revue internationale de psychosociologie et de gestion des comportements organisationnels, 29(48), 27-43.

Ruiz, E., Brion, S. et Parmentier, G. (2015). Les barrières à adoption du crowdsourcing pour innover. STAIMS 3 : créativité et organisation, quels enjeux en management stratégique ? $14^{e}$ Conférence de l'Association internationale de management stratégique. Paris, 3-5 juin.

SpInuzzi, C. (2012). Working alone together : coworking as emergent collaborative activity. Journal of Business and Technical Communication, 26(4), 399-441.

Stevens, E. (2009). Cocréation de valeur et communautés d'utilisateurs : vers un renouvellement des modèles de chaine de valeur et d'innovation. Revue management \& avenir, 8(28), 230-244.

Suire, R. (2016). La performance des lieux de cocréation de connaissances. Le cas des fablabs. Réseaux, 2(196), 81-109.

Tirole, J. (2016). Économie du bien commun. Paris, Presses universitaires de France.

Trudel, L., Simard, C. et Vonarx, N. (2007). La recherche qualitative est-elle nécessairement exploratoire? Recherches Qualitatives, (5), 38-45.

VAast, E. (2002). Les communautés de pratique sont-elles pertinentes. $11^{e}$ Conférence de l'Association internationale de management stratégique. Paris, France, 5-7 juin. 
Von Hippel, E. (1986). Lead users : a source of novel product concept. Management sciences, 32(7), 791-805.

WENGER, E. (1998). Communities of practice : learning as a social system. Systems Thinker, juin.

Zacchiroli, S. (2014). « Logiciel libre, Cours 8 - Development ». Support de cours, Laboratoire PPS, Université Paris Diderot. Récupéré le 17 avril 2017 du site : http://upsilon.cc/zack/teaching/1314/freesoftware/. 